\title{
A phase II study of lapatinib and bevacizumab as treatment for HER2-overexpressing metastatic breast cancer
}

\author{
Hope S. Rugo • A. Jo Chien · Sandra X. Franco • Alison T. Stopeck • \\ Alexa Glencer - Soumi Lahiri $\cdot$ Michael C. Arbushites $\cdot$ Janet Scott $\cdot$ \\ John W. Park · Clifford Hudis • Ben Nulsen · Maura N. Dickler
}

Received: 7 December 2011/Accepted: 9 December 2011/Published online: 24 December 2011

(C) The Author(s) 2011. This article is published with open access at Springerlink.com

\begin{abstract}
Preclinical data have demonstrated that the combination of antihuman epidermal growth factor receptor-2 (anti-HER2) and antivascular endothelial growth factor (anti-VEGF) - targeted agents has antitumor activity; these data indicate certain patients with HER2-overexpressing breast cancer may derive clinical benefit from this combination. The purpose of this single-arm phase II study was to determine the efficacy and safety of the dualtargeting combination of lapatinib and bevacizumab. Women with HER2-overexpressing advanced breast cancer received 1,500 mg oral lapatinib daily plus $10 \mathrm{mg} / \mathrm{kg}$ IV bevacizumab every 2 weeks. The primary endpoint was progression-free survival (PFS) at week 12; secondary endpoints included overall tumor response rate (ORR), clinical benefit rate (CBR), duration of response,
\end{abstract}

H. S. Rugo $(\bowtie) \cdot$ A. Jo Chien · A. Glencer · J. Scott .

J. W. Park

Helen Diller Family Comprehensive Cancer Center, University of California San Francisco, 1600 Divisadero St., 2nd Floor, Box 1710, B-608, San Francisco, CA 94143, USA

e-mail: hrugo@medicine.ucsf.edu

\section{S. X. Franco}

Breast Cancer Center, Memorial Cancer Institute, 3700 Johnson St., Hollywood, FL 33021, USA

\section{A. T. Stopeck \\ Arizona Cancer Center-North Campus, 3838 N. Campbell Ave., Tucson, AZ 85719, USA}

S. Lahiri - M. C. Arbushites

Medicine Development Centre Oncology, GlaxoSmithKline,

1250 S. Collegeville Rd., Collegeville, PA, USA

C. Hudis · B. Nulsen · M. N. Dickler

Memorial Sloan Kettering Cancer Center, 1275 York Ave.,

New York, NY, USA time-to-response, PFS, and safety. Circulating tumor cells (CTC) and circulating endothelial cells (CEC) were measured at baseline and during study treatment as potential response markers. Fifty-two patients with stage IV disease were enrolled. The 12-week investigator-assessed PFS rate was $69.2 \%$ (95\% confidence interval [CI]: 54.9, 81.3). Median PFS was 24.7 weeks (95\% CI: 20.4, 35.1), and the CBR was $30.8 \%$ (95\% CI: 18.7, 45.1). Of 45 patients with measurable disease, 6 were determined to have a partial response per Response Evaluation Criteria in Solid Tumors (ORR: 13.3\%; 95\% CI: 5.1, 26.8). The most common adverse events (AEs) included diarrhea, rash, and fatigue; most of these were either grade 1 or 2. Clinical responses were correlated with decreases in CTC and CEC. Lapatinib plus bevacizumab was active in patients with HER2overexpressing breast cancer. The AE profile of the combination was consistent with the known profiles for these agents.

Keywords HER2 - Tyrosine kinase inhibitor . Targeted therapy · Breast cancer - Lapatinib . Bevacizumab · VEGF

\section{Introduction}

Approximately $20-30 \%$ of all breast cancers overexpress the human epidermal growth factor receptor-2 (HER2) oncoprotein $[1,2]$. Although the prognosis for early stage HER2-overexpressing cancers has improved dramatically with the use of the monoclonal antibody trastuzumab in the adjuvant setting, both acquired and intrinsic resistance limit effective treatment of this highly aggressive subset of breast cancer in the metastatic setting [3, 4]. A minority (15-26\%) of patients with HER2-overexpressing metastatic tumors 
respond to first-line trastuzumab monotherapy, and most initial responders will develop disease progression within 1 year $[5,6]$. Therefore, novel and rational strategies targeting HER2-driven breast cancers are needed.

Of the identified angiogenic factors, vascular endothelial growth factor (VEGF) is the most potent and specific regulator of both normal and pathologic angiogenesis [7]. In preclinical studies, HER2-overexpression was associated with upregulation of VEGF in human breast cancer cell lines [8], and increased VEGF RNA and protein expression in human breast cancer xenograft models [9]. Moreover, in a large clinical cohort, patients whose tumors overexpressed HER2 and upregulated VEGF had worse survival compared with patients whose tumors overexpressed HER2 but not VEGF [10]. These data raise the possibility that aggressive phenotypes of HER2overexpressing breast cancers may be mediated by VEGF and provide a rationale for combining anti-HER2 and anti-VEGF therapies in the treatment of HER2-overexpressing breast cancers.

In HER2-overexpressing human breast cancer xenografts, the combination of trastuzumab and bevacizumab, an anti-VEGF-A monoclonal antibody, reduced xenograft tumor volume compared with single-agent controls [9]. A phase II study presented at the San Antonio Breast Cancer Symposium in 2009 treated 50 patients with HER2-overexpressing metastatic breast cancer (MBC) in the first-line setting with the combination of trastuzumab and bevacizumab, reported a clinical benefit rate (CBR) of $60 \%$, median time to progression (TTP) of 7.1 months, and a median overall survival (OS) of 43.8 months [11].

Lapatinib is a potent and specific reversible small molecule dual tyrosine kinase inhibitor (TKI) of both HER2 and epidermal growth factor receptor (EGFR). In trastuzumab-refractory disease, lapatinib plus capecitabine improved response and TTP compared with capecitabine alone $[12,13]$. Among 140 patients who had not previously received chemotherapy or trastuzumab for HER2positive disease, lapatinib combined with pazopanib, a small-molecule angiogenesis inhibitor of the VEGF receptor (VEGFR), led to a 12-week progression-free survival (PFS) of $84.1 \%$ compared to $63 \%$ with lapatinib monotherapy. A subsequent analysis on combining higher doses of lapatinib and pazopanib revealed no difference in response rates compared with the lower-dose arm but demonstrated a significant increase in serious toxicity [14].

In this study, a novel approach that combined lapatinib with bevacizumab to treat advanced HER2-overexpressing breast cancer was examined. In addition, circulating tumor cells (CTC) and circulating endothelial cells (CEC) were measured at baseline and during study treatment as potential early markers of response.

\section{Methods}

Patients

This phase II open-label multicenter study (EGF103890, NCT00444535) enrolled women aged $\geq 18$ years with histologically confirmed locally advanced stage III/IV breast cancer that overexpressed HER2 (either $3+$ by immunohistochemistry [IHC] or positive fluorescence in situ hybridization [FISH+]). Patients were eligible if they had: an Eastern Cooperative Oncology Group (ECOG) performance status of 0 or 1 ; adequate hepatic, renal, and hematologic function; and a cardiac ejection fraction within the institutional normal range. Prior anticancer therapy for MBC, including prior HER2- and VEGFdirected, was permitted but not required. Patients with recent major surgery, wound healing difficulties, proteinuria, or significant vascular, cardiac, and/or bleeding disorders were excluded. Women of childbearing potential were required to have a negative serum pregnancy test at baseline and to use appropriate contraception. The institutional review board for each participating institution approved the study protocol. This study was conducted in accordance with good clinical practice, all applicable regulatory requirements, and the guiding principles of the Declaration of Helsinki. All patients provided written informed consent before enrollment.

\section{Study design and treatment plan}

Eligible patients received study treatment $(1,500 \mathrm{mg}$ oral lapatinib daily plus $10 \mathrm{mg} / \mathrm{kg}$ bevacizumab IV every 2 weeks) until disease progression, withdrawal due to unacceptable toxicity, or withdrawal of consent. Dose delays of up to 2 weeks and/or dose reductions were allowed to manage toxicity. Lapatinib was discontinued in patients with symptomatic decreases in left ventricular ejection fraction (LVEF). Patients with asymptomatic LVEF decreases continued therapy and had a repeat evaluation within 2 weeks. If the abnormal LVEF decrease was confirmed, therapy was temporarily discontinued and could be restarted at a lower dose following event resolution.

The treatment safety profile was evaluated after the first 10 patients received 6 weeks of study treatment. If $>4$ doselimiting toxicities (DLTs) were reported, the dose of lapatinib was reduced by $500 \mathrm{mg}$ per day and the combination was reevaluated in ten additional patients. If $\leq 3$ DLTs were observed, the study was opened to enrollment at full dose.

Study endpoints

Based on a reported 4-month PFS rate of $41 \%$ among trastuzumab-refractory patients receiving single-agent 
lapatinib, and to mirror the design of the lapatinib and pazopanib combination trial, the primary endpoint of this study was PFS rate at week $12[15,16]$. Secondary endpoints included overall tumor response rate (ORR; complete or partial response confirmed $\geq 4$ weeks from first response), CBR (confirmed complete response [CR] + partial response $[\mathrm{PR}]$ or stable disease for $\geq 24$ weeks), duration of response, time to response, PFS, and safety. An exploratory analysis correlating baseline and on-treatment changes in CTCs and CECs with efficacy outcomes was also performed.

\section{Assessment of adverse events (AEs)}

AEs were graded according to the National Cancer Institute Common Toxicity Criteria, version 3.0. Patients were evaluated prior to each bevacizumab infusion and had a thorough physical exam. Echocardiograms or multiplegated acquisition scans were performed at week 8 and every 12 weeks thereafter. A cardiac event was defined as a symptomatic decline in LVEF, or an asymptomatic $\geq 20 \%$ decrease in LVEF below the institution's lower limit of normal.

\section{CTC and CEC analysis}

Blood for CTC and CEC analysis was obtained at: baseline: weeks $2,6,8$, and 12; and every 12 weeks until the end of study treatment. CTCs were measured by 2 independent methods: CellSearch ${ }^{\circledR}$ (Veridex, LLC, Raritan, NJ) [17] and immunomagnetic enrichment followed by flow cytometry (IE/FC) [18]. In brief, for IE/FC, cells were analyzed using IE with anti-EpCAM antibody-conjugated iron oxide nanoparticles followed by fluorescence-activated cell sorting (FACS) analysis for quantification. CTC were defined as EpCAM-positive, CD45-negative, and nucleated.

CEC were evaluated using a modified FC assay [19]. Following red blood cell lysis, CEC-related cell populations were identified by 4-color FC using a FACSCalibur ${ }^{\text {TM }}$ flow cytometer (BD Biosciences, San Jose, CA). CECs were defined as CD34-positive and leukocyte common antigen (CD45)-negative. In addition, total CECs were defined as CD31-positive or CD146-positive and progenitor CEC as CD31-positive and CD133-positive.

\section{Statistical analyses}

The study was designed to lead to a decision between 2 prespecified hypotheses on the probability $(P)$ of the 12-week PFS rate. The null hypothesis (i.e., $H_{0}: P=40 \%$ ) was considered to reflect a PFS rate that was not a clinically significant benefit over existing therapies. The alternative hypothesis (i.e., $H_{\mathrm{A}}: P=60 \%$ ) was considered to be a clinically significant improvement. Enrollment of 52 patients provided $84 \%$ power to evaluate these hypotheses using a 1-sided exact binomial test procedure. A minimum of 28 patients (factoring an additional $3 \%$ for a 1-sided type I error) were required to reach week 12 without disease progression to reject $H_{0}$.

All efficacy analyses were conducted on the intent-totreat population. Efficacy analyses were based on the investigator's evaluation of disease according to Response Evaluation Criteria in Solid Tumors (RECIST) [20]. The primary endpoint, 12-week PFS rate, was defined as the percentage of patients with no evidence of disease progression or death due to any cause for a minimum of 77 days. Patients with unknown or missing responses at week 12 or withdrawing from treatment by week 12 due to an $\mathrm{AE}$ were considered progressors. Patients with unknown or missing responses at week 12 followed by a nonprogressive disease response were evaluated based on response. PFS was defined as time from randomization until disease progression or death due to any reason.

CTC and CEC results were correlated to CBR and PFS. CTCs, measured using the Veridex and IE/FC methods, were tested for correlation at each time point using Spearman's test. CTC and CEC values (at each time point and change from baseline) were tested for association with CBR using the Wilcoxon rank-sum test. For correlation with PFS, Cox proportional hazards models were used. In the PFS models, CTC values at baseline, week 2 , and weeks $6-8$ were dichotomized into $(0,1)$ variables using the accepted cutoff values for CellSearch (cells/ $7.5 \mathrm{~mL}=5$ ); IE/FC and endothelial cells were modeled using cell counts as continuous variables (log scale). Additional PFS models evaluated change from baseline for CTC and CEC as a dichotomous variable defined as increase or no increase at each time point.

\section{Results}

Dose evaluation cohort

The first 10 patients were enrolled between March 2007 and May 2007. A total of 54 AEs were reported; 53 (98\%) were grade 1 or 2 . As the initial 10-patient cohort had only 1 DLT (grade 3 skin rash with grade 1 fever), the study was opened to full recruitment.

Study population

This trial enrolled 52 patients between March 2007 and April 2008 (Table 1). All patients had stage IV disease, and 51 of these patients were either FISH-positive or IHC 3+ for HER2-overexpression. Most patients had received prior 
Table 1 Patient characteristics

\begin{tabular}{ll}
\hline & $\begin{array}{l}\text { Lapatinib plus } \\
\text { bevacizumab } \\
(N=52)\end{array}$ \\
\hline Median age, years & 52.0 \\
Race, $n(\%)$ & \\
White & $42(81)$ \\
African American & $7(13)$ \\
Asian & $3(6)$ \\
Stage IV, $n(\%)$ & $52(100)$ \\
HER2 FISH+ or IHC $3+($ local $), n(\%)$ & $51(98)^{\mathrm{a}}$ \\
ECOG 0/1, $n$ (\%) & $28(54) / 24(46)$ \\
Visceral \pm nonvisceral sites, $n(\%)$ & $43(83)$ \\
ER/PR status, $n$ (\%) & \\
ER-/PR- & $26(50)$ \\
ER+/PR any & $26(50)$ \\
Prior chemotherapy regimens & $3(0-12)$ \\
in the metastatic setting, $n$ (range) & \\
Prior trastuzumab therapy, $n(\%)$ & $47(90)$ \\
Neoadjuvant & $2(4)$ \\
Adjuvant & $7(13)$ \\
Metastatic & $42(89)$ \\
Median duration of prior & $84.1(5.3-434.3)$ \\
trastuzumab, weeks (range) & \\
Neoadjuvant & $8.1(2.1-14.0)$ \\
Adjuvant & $12.0(6.1-151.0)$ \\
Metastatic & $84.1(5.3-434.3)$ \\
Prior lapatinib in the metastatic & $11(21)$ \\
setting, $n(\%)$ & $30.7(3.3-52.3)$ \\
Median treatment duration, & \\
weeks (range) & \\
Prior hormonal therapy, $n(\%)$ & \\
\hline
\end{tabular}

HER2 human epidermal growth factor receptor-2, FISH+ fluorescence in situ hybridization-positive, IHC immunohistochemistry, $E C O G$ Eastern cooperative oncology group, $E R$ - estrogen receptornegative, $P R$ - progesterone receptor-negative, $E R+$ estrogen receptorpositive

${ }^{\text {a }}$ FISH results not available for one IHC $1+$ patient who received $>2$ years of prior trastuzumab and lapatinib; this patient progressed on day 38

chemotherapy (96\%) and/or prior trastuzumab (90\%). These patients were considered heavily pretreated, with a median of 3 prior chemotherapy regimens (range 0-12) for metastatic disease as well as a median prior exposure of 30.7 weeks to lapatinib (range 3.3-52.3; Table 1) and 84.1 weeks to trastuzumab in the metastatic setting (range 5.3-434.3; Table 1).

Delivered therapy and compliance

Lapatinib was generally well tolerated (mean daily dose, $1,497 \mathrm{mg} / \mathrm{d})$ with dose reductions to $1,250 \mathrm{mg} / \mathrm{d}$ in $3(6 \%)$
Table 2 Summary of clinical efficacy

\begin{tabular}{ll}
\hline & $\begin{array}{l}\text { Lapatinib plus } \\
\text { bevacizumab } \\
(N=52)\end{array}$ \\
\hline PFS rate at week 12 & $36(69)$ \\
Patients reaching week 12 without & \\
$\quad$ PD $n(\%)$ & $69.2(54.9,81.3)$ \\
Crude 12 -week PFS rate, & \\
$\%$ (95\% CI) & $13.3(5.1,26.8)$ \\
ORR & \\
CR or PR confirmed, \% (95\% CI) & $30.8(18.7,45.1)$ \\
CBR & \\
CR or PR or SD $\geq 24$ weeks, & \\
$\%(95 \%$ CI) & $27(52)$ \\
Overall PFS & $25(48)$ \\
Progressions $n(\%)$ & $24.7(20.4,35.1)$ \\
Censored $n(\%)$ & \\
Median PFS, weeks, &
\end{tabular}

$P F S$ progression-free survival, $P D$ progressive disease, $C I$ confidence interval, $O R R$ overall response rate, $C R$ complete response, $P R$ partial response, $C B R$ clinical benefit rate, $S D$ stable disease

a Patients who did not die or progress until the clinical cutoff for these data (July 22, 2008)

patients and to $1,000 \mathrm{mg} / \mathrm{d}$ in $2(4 \%)$ patients for toxicity. Lapatinib was withheld for $\geq 24 \mathrm{~h}$ in 11 patients and bevacizumab was delayed in 8 patients, most frequently to manage diarrhea and hypertension, respectively.

\section{Clinical AEs}

The most common AEs were diarrhea, rash, fatigue, nausea, headache, and epistaxis, reflecting the known safety profile of both drugs (Table 2); most events were either grade 1 or 2 . AEs led to treatment discontinuation in 5 (10\%) patients: grade 3 rash with grade 1 fever; grade 3 alanine aminotransferase/aspartate aminotransferase (ALT/ AST) elevation; grade 2 left ventricular dysfunction; grade 3 hypertension; and grade 2 anorexia with grade 2 fatigue, and grade 1 nausea.

One patient reported grade 3 diarrhea that lasted 3 days; lapatinib was withheld for 6 days and restarted at $1,250 \mathrm{mg} / \mathrm{d}$ without recurrent symptoms.

Two patients reported grade 3 rash. The first patient developed an extensive grade 3 rash accompanied by a grade 1 fever after 10 days of study treatment. Study treatment was discontinued and the rash resolved with oral steroids. The second patient experienced a grade 3 rash after 19 days of study treatment; lapatinib was withheld then restarted at $1,250 \mathrm{mg} / \mathrm{d}$ on day 33 . The patient withdrew from the study on day 40 for personal reasons. 
Five $(10 \%)$ patients experienced a grade 1 or 2 decrease in LVEF; 1 patient discontinued study treatment due to a grade 2 decline in LVEF. Two of these events coincided with disease progression; 1 coincided with a viral syndrome that led to treatment withdrawal; and the remaining 2 events resolved at the next evaluation. All 5 patients had received prior anthracyclines and trastuzumab.

Grade 3 ALT/AST elevation was reported in 1 patient after 127 days on study. Study treatment was withheld on day 136 , restarted at full dose on day 141 , and dosereduced to $1,000 \mathrm{mg} / \mathrm{d}$ on day 153 . The elevation persisted and the patient was permanently withdrawn from study on day 169 . Grade 4 hyperbilirubinemia was reported in 1 patient 2 weeks after treatment discontinuation due to disease progression.

Hypertension was reported in 12 (23\%) patients; 10 were grade 1 or 2 , and 2 cases were grade 3 leading to treatment withdrawal in 1 patient.

Grade 4 hydronephrosis was reported in 1 patient after 148 days of study treatment attributed to disease progression. Grade 3 gastrointestinal hemorrhage, gastritis, and anemia was reported in 1 patient 10 days after treatment withdrawal due to disease progression.

\section{Analysis of efficacy outcomes}

The investigator-assessed 12-week PFS rate was $69.2 \%$ based on 36 patients not progressing by week 12 . The median PFS was 24.7 weeks, and the CBR was $30.8 \%$. Of 45 patients with measurable disease, 6 were determined to have a PR per RECIST (ORR: 13.3\%; Table 2). Five patients responded by week 6; the sixth patient responded by week 12 . Responses were durable, lasting 43, 120, 127, 191, 247, and 455 days; responses of 43, 127, and 455 days were ongoing and censored at the last radiology assessment before reporting (Fig. 1).

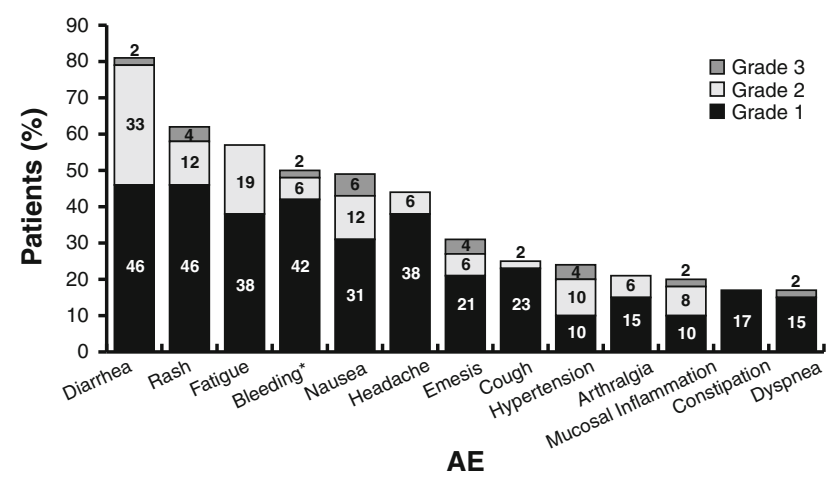

Fig. 1 AEs in $>15 \%$ of patients. *Bleeding includes epistaxis $(40 \%)$, gingival bleeding (4\%), gastric hemorrhage and lower gastrointestinal hemorrhage (2\%), and wound hemorrhage (2\%). AEs adverse events
Correlative analysis of CTC and CECs

Correlation between Veridex and IE/FC was statistically significant at each time point, with $R$ values ranging from 0.37 to $0.42(P<.05 ; n=29-32)$. Low CTC levels by either assay were associated with $\mathrm{CBR}$ at weeks $6-8$ (CellSearch: $P=.023, n=38$; IE/FC: $P=.032, n=30$ ) but not at baseline or week 2. Compared with baseline, CTC decrease by IE/FC at weeks $6-8$ was associated with CBR $(P=.0098, n=28$; Fig. 2a).

Shorter PFS was observed in patients with CTC $\geq 5$ cells/7.5 mL by CellSearch (week $2, P=.0135, n=43$; Fig. $2 \mathrm{~b}$; weeks $6-8, P=.0036, n=38)$. Patients with high levels of CTC by IE/FC at weeks 6-8 were also found to have a shorter PFS $(P=.0088 ; n=34)$. An increase in CTC from baseline to weeks $6-8$ ( $>0$ vs. $\leq 0$ ) by either assay was associated with decreased PFS (CellSearch: $P=.0033, n=34$; IE/FC: $P=.0179, n=28$ ).

Although change in total CEC did not correlate with CBR or PFS, a decrease in endothelial progenitor cells (CD-31-positive and CD133-positive) at weeks 6-8 was associated with $\mathrm{CBR}(P=.021, n=25)$.

\section{Discussion}

Although other trials have examined the combination of anti-HER2 and anti-VEGF agents in HER2-driven breast cancer treatment, this study is the first to report results on an oral TKI and a monoclonal antibody. Moreover, this is the only phase II study to describe combination therapy in a heavily pretreated population. In this report, daily lapatinib plus bevacizumab resulted in a 12-week PFS rate of $69.2 \%$, and a CBR and ORR of 30.8 and $13.3 \%$, respectively. Many observed responses were durable and the median PFS was 24.7 weeks. These data are provocative considering that $90 \%$ of the patients received a median of 84.1 weeks of prior trastuzumab in any setting.

Other studies have also shown promising activity with a combined anti-HER2 and anti-VEGF approach, although in the trastuzumab-naive first-line metastatic setting, making direct comparisons with the present trial is difficult. Hurvitz et al. [11] reported final results of a phase II trial of 50 patients with locally recurrent or metastatic HER2-overexpressing breast cancer receiving trastuzumab plus bevacizumab in the first-line metastatic setting that demonstrated an impressive CBR of $60 \%$ and a median 9.2month TTP. Furthermore, a phase II trial investigating lapatinib and pazopanib randomized 1 cohort of patients to lapatinib versus lapatinib plus pazopanib as first-line therapy for HER2-overexpressing MBC; a second cohort received a higher dose of pazopanib combined with lapatinib without randomization. An analysis of the first cohort showed an 
Fig. 2 a Decrease in CTC at weeks 6-8 compared with baseline by IE/FC is associated with clinical benefit. b CTC $<5$ / $7.5 \mathrm{~mL}$ by CellSearch ${ }^{\circledR}$ at week 2 is associated with improved PFS. CTC circulating tumor cells, $I E / F C$ immunomagnetic enrichment and flow cytometry, $P F S$ progression-free survival, $H R$ hazard ratio, $C I$ confident interval, $T x$ treatment

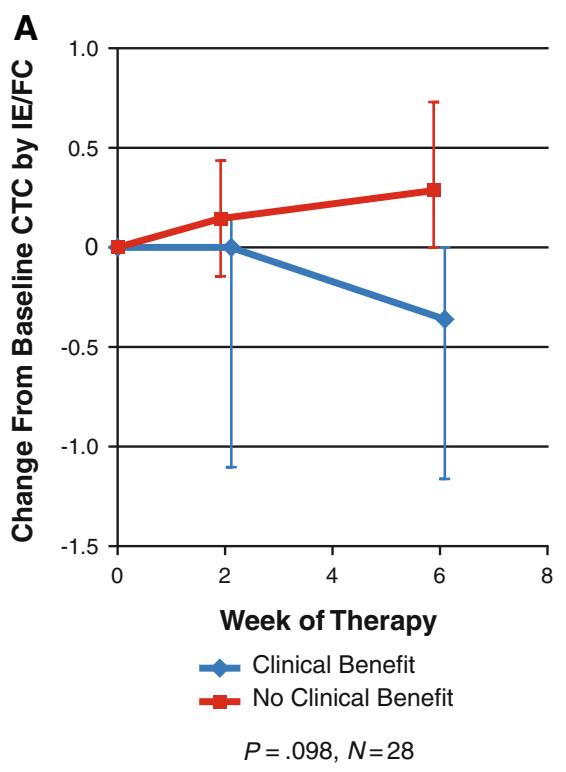

improved response rate with the combination (36\%) compared with lapatinib alone (22\%); however, toxicities were noted when pazopanib was given at a higher dose [14].

In this trial, changes in CTCs, as measured by two independent assays, correlated with both CBR and PFS. These data confirm previous observations showing that CTC fluctuations in patients receiving systemic treatment for MBC are predictive of outcome [17, 21-23]. The 2 different methodologies showed relatively weak but statistically significant correlation with each other, suggesting that the 2 techniques are capable of recognizing overlapping cell populations. As the IE/FC method allows CTC isolation for molecular analysis, continued validation of this technique is of interest for future research.

Reproducible, reliable, and easily obtainable markers for response and resistance to antiangiogenic therapy are needed. This study demonstrates that in patients with HER2-positive disease who received bevacizumab in combination with lapatinib, CBR was associated with a decrease in CD133-positive CECs. This finding supports previous data in patients with $\mathrm{MBC}$ receiving bevacizumab and erlotinib that showed the magnitude of change in total CECs from weeks $0-3$ predicted response at first assessment $(P=.018)$ [24]. CECs have been evaluated primarily as markers of angiogenesis; controversy exists regarding methodology, which cannot be addressed in the context of this small trial. Further validation of the role of CECs as early predictors of response in patients receiving antiangiogenic therapy is ongoing.

Clinical studies suggest that combined targeting of HER2 and VEGFR may be an effective strategy as treatment for HER2-overexpressing breast cancer but that toxicity may limit dose for some combinations. This approach may offer a new option for treating patients whose disease has progressed on or after trastuzumab and could be potentially used in an early stage setting to target intrinsically resistant disease. The phase III adjuvant BETH study comparing chemotherapy and trastuzumab to the same treatment with the addition of bevacizumab has completed accrual and is awaiting analysis [25]. ECOG 1105 evaluated a similar approach as first-line therapy for MBC but closed early due to poor accrual [26].

Lapatinib monotherapy in patients previously treated with trastuzumab in the advanced setting has shown limited efficacy $[15,27]$. In a recent phase II study randomizing patients with HER2-positive metastatic disease progressing on prior trastuzumab therapy to receive lapatinib versus lapatinib and trastuzumab, single-agent lapatinib led to a median PFS of 8.1 weeks and an ORR of 6.9\% [28]. Similarly, treatment with bevacizumab alone in HER2positive and HER2-negative disease has led to ORRs less than 7\% [29]. The present study results suggest that the combination of lapatinib and bevacizumab may be more effective than lapatinib or bevacizumab alone in this setting and may be a nonchemotherapy alternative for some patients. The combination of lapatinib and trastuzumab also demonstrated improved efficacy compared with trastuzumab alone in the trial noted above [28]. Other nonchemotherapy-based strategies for treating HER2overexpressing tumors that progress on trastuzumab are being explored in ongoing clinical trials with encouraging results to date, including trastuzumab plus pertuzumab (a novel HER2 antibody) and the targeted immunotherapy trastuzumab DM-1 [30, 31].

Finding effective therapies for patients with resistant or refractory HER2-overexpressing breast cancer remains an 
important therapeutic goal. The combination of lapatinib and bevacizumab is a promising approach to the treatment of HER2-overexpressing disease and warrants further investigation in combination with chemotherapy in advanced disease. Identifying reliable markers that predict both resistance to anti-HER2 agents and response to anti-VEGFR therapies is necessary to optimize this treatment strategy.

Acknowledgments This study was funded by GlaxoSmithKline (NCT00444535). All listed authors meet the criteria for authorship set forth by the International Committee of Medical Journal Editors. Editorial support in the form of editorial suggestions to draft versions of this article, assembling tables and figures, collating author comments, copyediting, fact checking, referencing, and graphic services was provided by Brad Imwalle, PhD, Antoinette Campo, and Tim Reilly at SCI Scientific Communications and Information. The authors would like to thank the patients who volunteered for this clinical trial and their families, friends, physicians, and research staff who cared for them.

Conflict of interest Hope S. Rugo: Genentech, GlaxoSmithKline, and Roche have provided research funding to the University of California San Francisco. A. Jo Chien, Sandra X. Franco, Alexa Glencer, Janet Scott, Clifford Hudis, and Ben Nulsen have no conflicts of interest to disclose. Alison T. Stopeck serves as a consultant and/or an advisor for Genentech. Soumi Lahiri has received remuneration from GlaxoSmithKline. Michael C. Arbushites has received remuneration from, and holds stock in, GlaxoSmithKline. John W. Park has received remuneration from Genentech and Roche. Maura N. Dickler serves as a consultant and/or advisor for Genentech, GlaxoSmithKline, and Roche.

Open Access This article is distributed under the terms of the Creative Commons Attribution Noncommercial License which permits any noncommercial use, distribution, and reproduction in any medium, provided the original author(s) and source are credited.

\section{References}

1. Slamon DJ, Godolphin W, Jones LA, Holt JA, Wong SG, Keith DE, Levin WJ, Stuart SG, Udove J, Ullrich A, Press MF (1989) Studies of the HER-2/neu proto-oncogene in human breast and ovarian cancer. Science 244:707-712

2. Slamon DJ, Clark GM, Wong SG, Levin WJ, Ullrich A, McGuire WL (1987) Human breast cancer: correlation of relapse and survival with amplification of the HER-2/neu oncogene. Science 235:177-182

3. Romond EH, Perez EA, Bryant J, Suman VJ, Geyer CE Jr, Davidson NE, Tan-Chiu E, Martino S, Paik S, Kaufman PA, Swain SM, Pisansky TM, Fehrenbacher L, Kutteh LA, Vogel VG, Visscher DW, Yothers G, Jenkins RB, Brown AM, Dakhil SR, Mamounas EP, Lingle WL, Klein PM, Ingle JN, Wolmark N (2005) Trastuzumab plus adjuvant chemotherapy for operable HER2-positive breast cancer. N Engl J Med 353:1673-1684

4. Piccart-Gebhart MJ, Procter M, Leyland-Jones B, Goldhirsch A, Untch M, Smith I, Gianni L, Baselga J, Bell R, Jackisch C, Cameron D, Dowsett M, Barrios CH, Steger G, Huang CS, Andersson M, Inbar M, Lichinitser M, Lang I, Nitz U, Iwata H, Thomssen C, Lohrisch C, Suter TM, Ruschoff J, Suto T, Greatorex V, Ward C, Straehle C, McFadden E, Dolci MS, Gelber RD, for the Herceptin Adjuvant (HERA) Trial Study Team (2005)
Trastuzumab after adjuvant chemotherapy in HER2-positive breast cancer. N Engl J Med 353:1659-1672

5. Valabrega G, Montemurro F, Aglietta M (2007) Trastuzumab: mechanism of action, resistance and future perspectives in HER2overexpressing breast cancer. Ann Oncol 18:977-984

6. Vogel CL, Cobleigh MA, Tripathy D, Gutheil JC, Harris LN, Fehrenbacher L, Slamon DJ, Murphy M, Novotny WF, Burchmore M, Shak S, Stewart SJ, Press M (2002) Efficacy and safety of trastuzumab as a single agent in first-line treatment of HER2overexpressing metastatic breast cancer. J Clin Oncol 20:719-726

7. Ferrara N, vis-Smyth T (1997) The biology of vascular endothelial growth factor. Endocr Rev 18:4-25

8. Yen L, You XL, Al Moustafa AE, Batist G, Hynes NE, Mader S, Meloche S, Alaoui-Jamali MA (2000) Heregulin selectively upregulates vascular endothelial growth factor secretion in cancer cells and stimulates angiogenesis. Oncogene 19:3460-3469

9. Epstein M, Ayala R, Tchekmedyian N, Borgstrom P, Pegram M, Slamon D (2002) HER2-overexpressing human breast cancer xenografts exhibit increased angiogenic potential mediated by vascular endothelial growth lactate (VEGF). Breast Cancer Res Treat 76:S143 (Abstract 570)

10. Konecny GE, Meng YG, Untch M, Wang HJ, Bauerfeind I, Epstein M, Stieber P, Vernes JM, Gutierrez J, Hong K, Beryt M, Hepp H, Slamon DJ, Pegram MD (2004) Association between HER-2/neu and vascular endothelial growth factor expression predicts clinical outcome in primary breast cancer patients. Clin Cancer Res 10:1706-1716

11. Hurvitz SA, Pegram MD, Lin L-S, Chan DS, Allen HJ, Dichmann RA, Hagenstad CT, Barstis J, Hermann RC, Hu EH, Moroose RL, Thomas SP, Vogel CL, Ryba N, Elashoff D (2009) Final results of a phase II trial evaluating trastuzumab and bevacizumab as first line treatment of HER2-amplified advanced breast cancer. Cancer Res 69:854s

12. Cameron D, Casey M, Oliva C, Newstat B, Imwalle B, Geyer CE (2010) Lapatinib plus capecitabine in women with HER-2-positive advanced breast cancer: final survival analysis of a phase III randomized trial. Oncologist 15:924-934

13. Cameron D, Casey M, Press M, Lindquist D, Pienkowski T, Romieu CG, Chan S, Jagiello-Gruszfeld A, Kaufman B, Crown J, Chan A, Campone M, Viens P, Davidson N, Gorbounova V, Raats JI, Skarlos D, Newstat B, Roychowdhury D, Paoletti P, Oliva C, Rubin S, Stein S, Geyer CE (2008) A phase III randomized comparison of lapatinib plus capecitabine versus capecitabine alone in women with advanced breast cancer that has progressed on trastuzumab: updated efficacy and biomarker analyses. Breast Cancer Res Treat 112:533-543

14. Slamon DJ, Stemmer sM, Johnston S, Kim S, Durante M, Pandite LN, Roychowdhury DF, Goodman VL (2009) Phase 2 study of dual VEGF/HER2 blockade with pazopanib + lapatinib in patients with first-line HER2 positive advanced or metastatic (adv/met) breast cancer [abstract]. Cancer Res 69:4114

15. Blackwell KL, Pegram MD, Tan-Chiu E, Schwartzberg LS, Arbushites MC, Maltzman JD, Forster JK, Rubin SD, Stein SH, Burstein HJ (2009) Single-agent lapatinib for HER2-overexpressing advanced or metastatic breast cancer that progressed on first- or second-line trastuzumab-containing regimens. Ann Oncol 20:1026-1031

16. Slamon D, Gomez HL, Kabbinavar FF, Amit O, Richie M, Pandite L, Goodman V (2008) Randomized study of pazopanib + lapatinib vs. lapatinib alone in patients with HER2-positive advanced or metastatic breast cancer [abstract]. J Clin Oncol 26:2999-3005 (Abstract 1016)

17. Cristofanilli M, Budd GT, Ellis MJ, Stopeck A, Matera J, Miller MC, Reuben JM, Doyle GV, Allard WJ, Terstappen LW, Hayes 
DF (2004) Circulating tumor cells, disease progression, and survival in metastatic breast cancer. N Engl J Med 351:781-791

18. Garcia JA, Rosenberg JE, Weinberg V, Scott J, Frohlich M, Park JW, Small EJ (2007) Evaluation and significance of circulating epithelial cells in patients with hormone-refractory prostate cancer. BJU Int 99:519-524

19. Mancuso P, Burlini A, Pruneri G, Goldhirsch A, Martinelli G, Bertolini F (2001) Resting and activated endothelial cells are increased in the peripheral blood of cancer patients. Blood 97:3658-3661

20. Therasse P, Arbuck SG, Eisenhauer EA, Wanders J, Kaplan RS, Rubinstein L, Verweij J, Van GM, van Oosterom AT, Christian MC, Gwyther SG (2000) New guidelines to evaluate the response to treatment in solid tumors. J Natl Cancer Inst 92:205-216

21. Botteri E, Sandri MT, Bagnardi V, Munzone E, Zorzino L, Rotmensz N, Casadio C, Cassatella MC, Esposito A, Curigliano G, Salvatici M, Verri E, Adamoli L, Goldhirsch A, Nole F (2010) Modeling the relationship between circulating tumour cells number and prognosis of metastatic breast cancer. Breast Cancer Res Treat 122:211-217

22. Munzone E, Nolé F, Zorzino L, Medici M, Minchella I, Cassatella MC, Casadio C, Picozzi M, Adamoli L, Goldhirsch A, Sandri M (2008) Acquisition of HER-2/neu over-expression on circulating tumor cells (CTCs) in patients (pts) with advanced breast cancer $(\mathrm{ABC})$ during chemotherapy [abstract]. J Clin Oncol 26(suppl):11017

23. Nole F, Munzone E, Zorzino L, Minchella I, Salvatici M, Botteri E, Medici M, Verri E, Adamoli L, Rotmensz N, Goldhirsch A, Sandri MT (2008) Variation of circulating tumor cell levels during treatment of metastatic breast cancer: prognostic and therapeutic implications. Ann Oncol 19:891-897

24. Rugo HS, Dickler MN, Scott JH, Moore DH, Melisko M, Yeh BM, Caravelli J, Brogi E, Hudis C, Park JW (2005) Change in circulating endothelial cells (CEC) and tumor cells (CTC) in patients (pts) receiving bevacizumab and erlotinib for metastatic breast cancer (MBC) predicts stable disease at first evaluation [abstract]. J Clin Oncol 23:525
25. ClinicalTrials.gov Web Site (2011) BETH Study: Treatment of HER 2 positive breast cancer with chemotherapy plus trastuzumab vs chemotherapy plus trastuzumab plus bevacizumab. http://www.clinicaltrials.gov/ct2/show/NCT00625898. Accessed 17 Nov 2011

26. ClinicalTrials.gov Web Site (2011) First-line chemotherapy and trastuzumab with or without bevacizumab in treating patients with metastatic breast cancer that overexpresses HER-2/NEU. http://www.clinicaltrials.gov/ct2/show/NCT00520975. Accessed Nov 172011

27. Blackwell KL, Kaplan EH, Franco SX, Marcom PK, Maleski JE, Sorensen MJ, Berger MS (2004) A phase II, open-label, multicenter study of GW572016 in patients with trastuzumab-refractory metastatic breast cancer [abstract]. J Clin Oncol 22:3006

28. Blackwell KL, Burstein HJ, Storniolo AM, Rugo H, Sledge G, Koehler M, Ellis C, Casey M, Vukelja S, Bischoff J, Baselga J, O'Shaughnessy J (2010) Randomized study of lapatinib alone or in combination with trastuzumab in women with ErbB2-positive, trastuzumab-refractory metastatic breast cancer. J Clin Oncol 28:1124-1130

29. Cobleigh MA, Langmuir VK, Sledge GW, Miller KD, Haney L, Novotny WF, Reimann JD, Vassel A (2003) A phase I/II doseescalation trial of bevacizumab in previously treated metastatic breast cancer. Semin Oncol 30:117-124

30. Baselga J, Imadalou K, Paton V, Gray D, Swai S (2009) Efficacy, safety and tolerability of dual monoclonal antibody therapy with pertuzumab + trastuzumab in HER $2+$ metastatic breast cancer patients previously treated with trastuzumab [abstract]. Cancer Res 69:3138

31. Vogel CL, Burris HA, Limentani S, Borson R, O'Shaughnessy J, Vukelja S, Agresta S, Klencke B, Birkner M, Rugo H (2009) A phase II study of trastuzumab-DM1 (T-DM1), a HER2 antibodydrug conjugate (ADC), in patients (pts) with HER2 + metastatic breast cancer (MBC): final results [abstract]. J Clin Oncol 27:1198-1207 (Abstract 1017) 